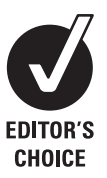

CHOICE

\title{
Vascular endothelial growth factor and angiopoietin-2 play a major role in the pathogenesis of vascular leakage in cirrhotic rats
}

\author{
P Melgar-Lesmes, ${ }^{1}$ S Tugues, ${ }^{1} \mathrm{~J}$ Ros, ${ }^{1}$ G Fernández-Varo, ${ }^{1}$ M Morales-Ruiz, ${ }^{1} \mathrm{~J}$ Rodés, ${ }^{2}$ \\ W Jiménez ${ }^{1,3}$
}

- Supplementary methods and a figure are published online only at http://gut.bmj.com/content/ vol58/issue2

${ }^{1}$ Biochemistry and Molecular Genetics Service, Institut $d^{\prime}$ Investigacions Biomèdiques August Pi i Sunyer (IDIBAPS), University of Barcelona,

Barcelona, Spain; ${ }^{2}$ Liver Unit, CIBERehd, Institut

d'Investigacions Biomèdiques August Pi i Sunyer (IDIBAPS),

University of Barcelona,

Barcelona, Spain; ${ }^{3}$ Department of Physiology I, Institut d'Investigacions Biomèdiques August Pi i Sunyer (IDIBAPS), University of Barcelona, Barcelona, Spain

Correspondence to: Dr W Jiménez, Servicio Bioquímica y Genética Molecular, Hospital Clinic Universitari, Villarroel 170, Barcelona 08036, Spain; wjimenez@clinic.ub.es

Revised 2 September 2008 Accepted 14 October 2008 Published Online First 31 October 2008

\section{ABSTRACT}

Background and aims: The extent and molecular mechanisms governing plasma extravasation and formation of ascites in cirrhosis are unknown. Vascular endothelial growth factor-A (VEGF-A) and angiopoietin-2 (Ang-2) are endogenous substances with powerful vascular permeability effects. We assessed regional blood flow, vascular leakage, mRNA and tissular expression of VEGF-A and Ang-2 and vascular permeability following VEGF receptor 2 blockade in control and cirrhotic rats to define the vascular territories showing altered vascular permeability in cirrhosis and to determine whether VEGFA and Ang-2 are involved in this phenomenon.

Methods: Arterial blood flow was analysed with the coloured microsphere method. Vascular leakage was measured and visualised with the dye Evan's Blue and colloidal carbon techniques, respectively. VEGF-A and Ang-2 expression were determined by real-time polymerase chain reaction (RT-PCR), immunohistochemistry and western blot. The effect on vascular permeability induced by VEGFR 2 blockade was assessed by administration of the receptor inhibitor SU11248.

Results: Arterial blood flow was increased in the mesentery, pancreas and small intestine but not in the kidney and spleen of cirrhotic rats as compared to controls. Increased vascular leakage was observed in the mesentery and liver, where colloidal carbon spread from microvessels to the adjacent fibrotic tracts. Increased hepatic and mesenteric expression of VEGF-A and Ang-2 was found in cirrhotic rats as compared to controls. Blockade of VEGFR 2 markedly reduced hepatic and mesenteric vascular leakage in cirrhotic rats.

Conclusions: Enhanced endothelial permeability is restricted to the hepatic and mesenteric vascular beds in cirrhotic rats with ascites and VEGF-A and Ang-2 are key factors in the signalling pathways regulating this dysfunction.

Ascites, the most common complication in patients with advanced liver disease, is invariably associated with portal hypertension and consists of the accumulation of liquid in the peritoneal cavity. ${ }^{1}$ Ascites in cirrhotic patients is mainly formed by salt and water, variable amounts of albumin and globulins, and a relatively low number of mesothelial cells and leucocytes. Because of this relatively simple composition the presence of ascites was classically considered to have little or no influence on the homeostasis of the peritoneal vasculature. Nevertheless, this has recently been challenged by several studies demonstrating that this liquid may possess vasodilator, proinflammatory and proangiogenic properties. ${ }^{2-7}$ However, whereas it is well documented that ascites in cirrhosis may behave as a bioactive fluid, the source of this liquid and the molecular gates regulating fluid extravasation in cirrhosis are less clear. Several pieces of circumstantial evidence point to the cirrhotic liver as an important source of ascites ${ }^{8}{ }^{9}$ but discussion still exists concerning the relative contribution of other organs located in the abdominal cavity, such as the intestine, spleen, pancreas or the mesenteric vasculature. On the other hand, vascular leakage depends on a number of variables including the physical properties of the fluid or the molecule being transported and the gradients between the intra- and the extravascular compartments. ${ }^{10}$ Moreover, transvascular transport may occur through channels or vesicles within individual cells or through inter-endothelial junctions between adjacent cells. ${ }^{11}$ Endothelial cells are the primary barrier to vascular extravasation, thus regulating the passage of molecules by a variety of mechanisms that are chiefly regulated by the initially called vascular permeability factor and now better known as the vascular endothelial growth factor type A (VEGF-A). This peptide belongs to the VEGF family which comprises five additional known members of structurally related dimeric glycoproteins: VEGF-B, VEGF-C, VEGF-D, VEGF-E and placental growth factor (PIGF). In addition, alternative exon splicing of the VEGF-A gene generates at least five VEGF isoforms, with 121, 145, 165, 189 or 206 amino acid residues with different bioavailability and with VEGF-A $\mathrm{A}_{121}$ and VEGF- $\mathrm{A}_{165}$ being the most frequently expressed forms. ${ }^{12}$ This family of growth factors binds and activates three VEGF transmembrane tyrosine kinase receptors: $\mathrm{VEGFR}_{1}$ (vascular endothelial growth factor receptor 1), $\mathrm{VEGFR}_{2}$ and $\mathrm{VEGFR}_{3}$, which differ in their ligand specificities. ${ }^{12}$ By interacting with the VEGFR 2 , VEGF-A not only induces angiogenesis but also disrupts vascular barrier function in diseased tissues. ${ }^{10}{ }^{11}$ The list of angiogenic substances that increase vascular permeability in vivo has recently been enlarged by angiopoietin-2 (Ang-2), a member of the angiopoietin family of proteins that dose-dependently induces oedema formation in the mouse paw assay. ${ }^{13}$ Angiopoietin-1 (Ang-1) is a Tie-2 receptor agonist that induces pericyte recruitment and maturation of blood capillaries. Then, capillary endothelial cells become quiescent and unresponsive to VEGF-A action unless Ang-2 is also secreted. Therefore, Ang-2 is a natural Ang-1 antagonist 
which displaces Ang-1 from its receptor, thereby disrupting Tie2 signalling. ${ }^{14}$ However, the contributory role of VEGF-A and Ang-2 to vascular leakage and oedema formation in cirrhosis is unknown. Identification of the source and molecular mechanisms governing vascular hyperpermeability in cirrhosis may potentially favour future strategies to reduce tissue injury in these patients.

\section{MATERIALS AND METHODS}

\section{Animals}

This study was performed in 38 male adult cirrhotic Wistar rats and in 23 control Wistar rats (Charles-River, Saint Aubin les Elseuf, France). Both groups were fed ad libitum with standard chow and distilled water containing phenobarbital. Cirrhosis was induced as described elsewhere. ${ }^{15}$ Cirrhotic rats without ascites were studied between 12 and 14 weeks and cirrhotic rats with ascites between 13 and 18 weeks. When present, ascites was tested for bacterial culture and was negative in all cirrhotic rats included in the study. Control rats were studied following a similar period of phenobarbital administration.

\section{Measurement of vascular permeability}

The dye Evan's Blue (EB; Sigma Chemical, St Louis, Missouri, USA) technique was used to measure capillary permeability to albumin. This technique is based on the principle that this dye binds avidly to the negatively charged intravascular albumin, therefore being a reliable estimate of transvascular fluxes of macromolecules. ${ }^{16}$ The dye was quantified by spectrophotometry at $620 \mathrm{~nm}$ and the results were calculated from a standard curve of Evan's Blue $(0.05-25 \mu \mathrm{g} / \mathrm{ml})$ and expressed as $\mu \mathrm{g} \mathrm{EB} / \mathrm{g}$ dry weight of tissue.

\section{Identification of hyperpermeable blood vessels of tissues and organs with colloidal carbon}

Colloidal carbon is a tracer that can be visualised in tissues by both macroscopy and high-resolution light microscopy. ${ }^{17}$ Control and cirrhotic rats with ascites were anaesthetised with ketamine $(50 \mathrm{mg} / \mathrm{kg})$ and injected intravenously (i.v.) with $2 \mathrm{ml} / \mathrm{kg}$ body weight of a 1:5 dilution (in PBS) of colloidal carbon (C11/1431A, Guenther Wagner, Hannover, Germany). One hour later, animals were killed by $\mathrm{CO}_{2}$ narcosis and liver specimens obtained from the middle lobe, the mesentery with the attached bowel, pancreas, spleen and right kidney were fixed in $4 \%$ formaldehyde. Liver, spleen and kidney were embedded in paraffin and processed for Giemsa staining. Tissue slides were visualised by high-resolution light microscopy (Olympus Bx51, Tokyo, Japan). Mesentery-bowel and pancreas were mounted under PBS immersion for viewing in a macroscope (MZ6; Leica Microsystems, Heerbrugg, Switzerland).

\section{Measurement of haemodynamic parameters \\ See supplementary data.}

mRNA expression of VEGF-A and Ang-2 in abdominal organs of cirrhotic rats

See supplementary data.

\section{Western blot of VEGF-A, Ang-1, Ang-2 and phosphorylated VEGFR $_{2}$ and immunohistochemistry of VEGF-A and Ang-2 See supplementary data.}

\section{Effect of VEGFR $\mathbf{2}_{\mathbf{2}}$ blockade on vascular permeability}

To test whether VEGFR 2 mediates vascular hyperpermeability in cirrhotic animals, 10 rats with cirrhosis and ascites were anaesthetised with inactine $(100 \mathrm{mg} / \mathrm{kg})$ and randomly received an oral administration of the VEGFR 2 antagonist SU11248 ( $\mathrm{n}=5 ; 20 \mathrm{mg} / \mathrm{kg}$ body weight) or vehicle ( $\mathrm{n}=5$; citratebuffered solution, pH 3.5). After $2.5 \mathrm{~h}$, vascular permeability was assessed in both groups of animals as described above and liver samples were frozen at $-80^{\circ} \mathrm{C}$ to further analyse $\mathrm{VEGFR}_{2}$ phosphorylation.

\section{Statistical analysis}

Data are expressed as mean with the SE. Statistical analysis of the results was performed by one-way analysis of variance (ANOVA) the Newman-Keuls test, and the unpaired Student $t$
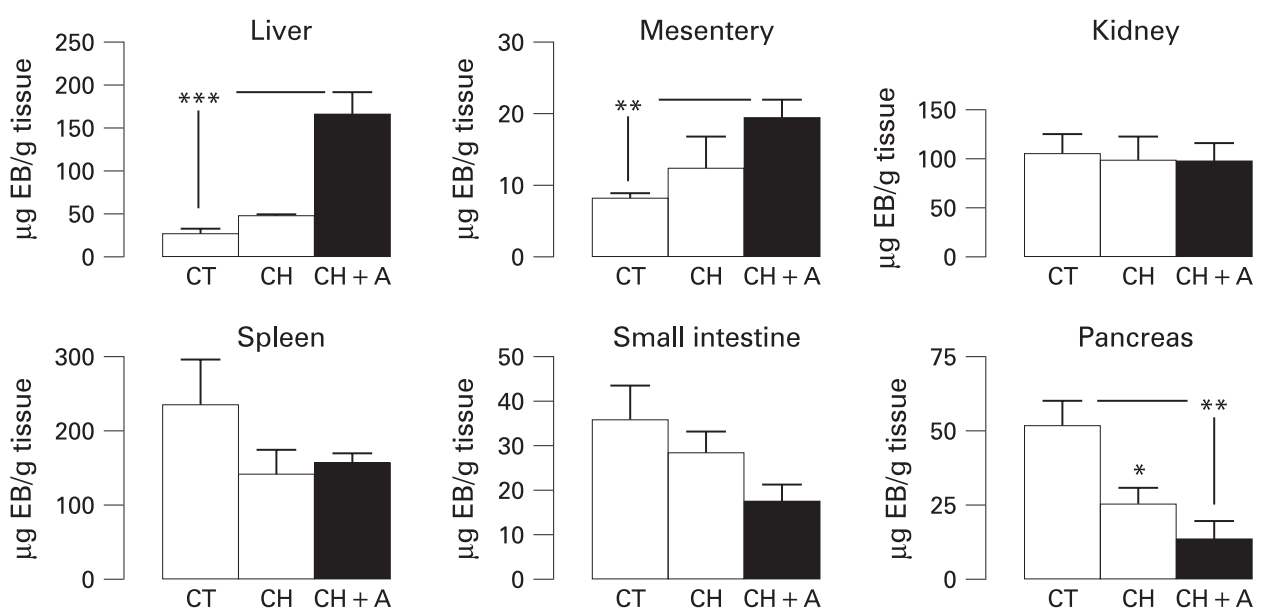

Figure 1 Measurement of vascular leakage in abdominal tissues with the Evan's Blue (EB) dye method. Animals were anaesthetised with ketamine $(50 \mathrm{mg} / \mathrm{kg})$ and injected in the penile vein with Evan's Blue $(30 \mathrm{mg} / \mathrm{kg})$ which was allowed to circulate for 30 min. The dye was washed out by perfusing the vascular tree with phosphate-buffered saline (PBS) containing heparin ( $50 \mathrm{U} / \mathrm{ml}$ ) for 5 min and then the vasculature was perfusion-fixed

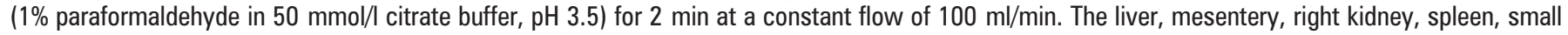
intestine and pancreas were obtained from control rats $(C T, n=8)$ cirrhotic rats without ascites $(\mathrm{CH}, \mathrm{n}=5)$ and cirrhotic rats with ascites $(\mathrm{CH}+\mathrm{A}$, $\mathrm{n}=8$ ) to assess the degree of vascular extravasation. The amount of the extracted dye was expressed as micrograms of Evan's Blue per gram of dry weight of each tissue. ${ }^{* *} p<0.001 ;{ }^{* *} p<0.01$ and ${ }^{*} p<0.05$ vs control rats (one-way ANOVA and Newman-Keuls tests). 
Figure 2 Giemsa-stained sections illustrating microvascular permeability in the liver of control and cirrhotic rats (upper panels). Low-power macrographs of leaky blood vessels of mesentery and pancreas of control and cirrhotic rats are also shown in the middle and lower panels, respectively. Colloidal carbon was injected intravenously and used as a tracer of local extravasation. Original magnifications: liver, $\times 200$; mesentery, $\times 10$; and pancreas, $\times 20$.



Control test when appropriate. Differences were considered to be significant at a $\mathrm{p}$ value of 0.05 or less.

\section{RESULTS}

\section{Vascular permeability in intra-abdominal tissues}

Basal leakage of Evan's Blue varied from one organ to other, ranging between 235.8 (SE 61.5) $\mu \mathrm{g} \mathrm{EB/g}$ tissue in the spleen and 8.3 (SE 0.8) $\mu \mathrm{g} \mathrm{EB/g}$ tissue in the mesentery. These fluctuations in basal permeability among the different organs are not uncommon and have been shown to depend on the degree of vascularisation of these organs. ${ }^{18}$ It is important to stress that the albumin-bound Evan's Blue remaining within the vascular compartment after tissue washout is considered to be negligible. $^{18}$ Figure 1 shows plasma extravasation in the liver, mesentery, kidney, spleen, small intestine and pancreas of control and cirrhotic rats, without and with ascites. A marked increase in plasma permeability was found in the liver and

Table 1 Systemic haemodynamics and arterial blood flow in the different organs in control and cirrhotic rats included in the haemodynamic protocol

\begin{tabular}{|c|c|c|}
\hline & Control rats $(n=7)$ & Cirrhotic rats $(n=7)$ \\
\hline \multicolumn{3}{|l|}{ Systemic haemodynamics } \\
\hline Mean arterial pressure $(\mathrm{mm} \mathrm{Hg})$ & $125(1)$ & $102(1)^{*}$ \\
\hline Heart rate (beats/min) & $371(5)$ & 374 (9) \\
\hline Cardiac index $(\mathrm{ml} / \mathrm{min} / 100 \mathrm{~g})$ & $21.9(0.5)$ & $40.8(2.9)^{* *}$ \\
\hline Systemic vascular resistance ( $\mathrm{mm} \mathrm{Hg} \mathrm{min/ml)}$ & $5.72(0.16)$ & $2.59(0.20)^{* *}$ \\
\hline \multicolumn{3}{|l|}{ Regional blood flow $(\mathrm{ml} / \mathrm{min} / 100 \mathrm{~g})$} \\
\hline Liver & $34.5(5.2)$ & $31.4(5.4)$ \\
\hline Mesentery & $18.2(2.8)$ & $53.3(10.6)^{*}$ \\
\hline Pancreas & $59.3(5.3)$ & $160.3(13 .)^{* *}$ \\
\hline Spleen & $123.5(18.4)$ & $123.4(16.6)$ \\
\hline Small intestine & $81.8(13.5)$ & $235.7(40.0)^{*}$ \\
\hline Kidney & $340.5(43.6)$ & $455.6(36.4)$ \\
\hline
\end{tabular}

Results are given as mean (standard error).

${ }^{*} p<0.01$ and ${ }^{* *} p<0.001$ vs control rats. 
mesentery of cirrhotic rats with ascites as compared to controls, with the differences between the two groups being 7-fold and more than 2-fold higher, respectively. No differences in this parameter were observed between the kidneys of cirrhotic and control animals. In contrast, spleen, small intestine and pancreas of rats with cirrhosis and ascites showed a tendency towards decreased capillary permeability in comparison to control rats, although differences only reached statistical significance in the pancreas. In fact, extravasation of Evan's Blue in the pancreas of cirrhotic rats was around four times lower than that in controls (fig 1). No differences were found in vessel permeability between control animals and cirrhotic rats without ascites in any of the organs in which this parameter was assessed, with the exception of the pancreas in which vessel permeability was reduced in comparison to control rats.

\section{Identification of hyperpermeable blood vessels using colloidal carbon}

Colloidal carbon is a precise marker for identifying leaky blood vessels at the ultrastructural level. ${ }^{19}$ Colloidal carbon particles are able to penetrate hyperpermeable endothelium but are retained by the underlying basal lamina if it is intact and continuous. Therefore, vascular leakage can be identified by deposits of colloidal carbon in the vascular basal lamina. No significant differences were found in the labelling pattern of the spleen, small intestine and right kidney between control and cirrhotic animals (data not shown). In contrast, cirrhotic animals displayed an altered distribution pattern of the tracer in the liver, mesentery and pancreas as compared to control rats. Figure 2 shows representative hepatic sections from a control rat and a cirrhotic rat with ascites. Whereas in control rats colloidal carbon was uniformly distributed over the liver parenchyma and spread from the portal space towards the hepatic sinusoids, in cirrhotic animals the presence of the tracer was more abundant and its distribution did not follow a regular pattern. It is of note that colloidal carbon was extensively located close to or within the fibrous septa.

Paralleling the results obtained at quantifying the mesenteric permeability, mesenteric vessels of cirrhotic rats became markedly leaky to colloidal carbon. In these animals the tracer escaped to the basal lamina, while in normal rats carbon particles were practically all retained in the interior of the mesenteric vasculature (fig 2). Thus, hepatic dysfunction is also associated with increased endothelial fenestration in the mesenteric vascular bed.

A final remarkable observation of this protocol was that the abnormal colloidal carbon distribution pattern found in the pancreas of cirrhotic rats followed an opposite direction to that observed in the liver and mesentery of these animals. As shown in fig 2, pancreatic vessels of control rats are highly permeable to the tracer, which results in the identification of a dense vascular network of capillaries. By contrast, this almost disappears on analysing the pancreatic tissue of cirrhotic animals.

\section{Systemic haemodynamics and arterial blood flow}

Since changes in blood flow may modulate vascular permeability ${ }^{20}$ we next assessed systemic haemodynamics and arterial blood flow in cirrhotic and control rats. As anticipated, cirrhotic rats displayed an important hypercirculatory syndrome characterised by arterial hypotension, high cardiac index and markedly reduced systemic vascular resistance (table 1).

Moreover, cirrhotic rats also showed a significantly increased arterial blood flow in the mesentery, pancreas and small intestine while no differences were registered in the spleen, kidney and liver. It should be noted, however, that total liver blood flow has a dual component: the arterial blood flow and the portal venous inflow, this latter parameter not being measured when using the microsphere method to assess blood flow. Since several studies have previously demonstrated an enhanced portal venous inflow in humans and rats with advanced liver disease, ${ }^{21}{ }^{22}$ it is very likely that the total hepatic blood flow was increased in our cirrhotic animals.

\section{mRNA expression of VEGF-A and Ang-2 in intra-abdominal tissues}

Since VEGF-A and Ang-2 are two major regulators of microvessel permeability, we next assessed whether organ permeability dysregulations are associated with altered expression of these messengers. As shown in table 2, changes in mRNA expression of VEGF-A and Ang-2 followed a parallel pattern to that observed in the analysis of regional alterations of vascular permeability.

These transcripts were significantly enhanced in the liver and mesentery of cirrhotic animals as compared to controls. In contrast, a marked reduction in VEGF-A and Ang-2 mRNAs was found in the pancreas of cirrhotic rats as compared to controls. Interestingly, a highly significant increase in Ang-2 mRNA, but not VEGF-A mRNA, was observed in the small intestine of cirrhotic animals compared to control animals. Finally, no differences in mRNA levels were observed between spleen and kidney of cirrhotic and control animals.

\section{VEGF-A and Ang-2 protein expression in the organs with altered vascular permeability}

Western blot analysis for VEGF-A in the liver showed two bands at $38 \mathrm{kDa}$ and $45 \mathrm{kDa}$ corresponding to the VEGF 121 and $\mathrm{VEGF}_{165}$ isoforms, respectively (fig 3 ). Densitometric analysis revealed increased VEGF-A protein content in cirrhotic livers as compared to controls, which was due to a rise in the $\mathrm{VEGF}_{165}$

Table 2 Quantitative real-time polymerase chain reaction of vascular endothelial growth factor- $A$ and angiopoietin-2 in abdominal tissues of control and cirrhotic rats with ascites

\begin{tabular}{llllll}
\hline \multirow{2}{*}{ Tissue } & \multicolumn{2}{l}{ Vascular endothelial growth factor- } & & Angiopoietin-2 & \\
\cline { 2 - 3 } \cline { 5 - 6 } \cline { 5 - 6 } & Control $(\mathbf{n}=\mathbf{5})$ & Cirrhosis $(\mathbf{n}=\mathbf{5})$ & & Control $(\mathbf{n}=\mathbf{5})$ & Cirrhosis $(\mathbf{n}=\mathbf{5})$ \\
\hline Liver & $1.14(0.2)$ & $1.91(0.2)^{*}$ & & $1.17(0.2)$ & \\
Mesentery & $1.09(0.2)$ & $5.59(0.8)^{*}$ & & $1.09(0.1)$ & $2.41(0.4)^{*}$ \\
Small intestine & $1.07(0.1)$ & $1.55(0.4)$ & & $1.17(0.1)$ & $3.58(0.6)^{*}$ \\
Pancreas & $1.04(0.2)$ & $0.53(0.1)^{*}$ & & $1.17(0.2)$ & $4.36(0.6)^{* *}$ \\
Spleen & $1.07(0.2)$ & $0.89(0.2)$ & & $1.07(0.2)$ & $0.57(0.1)^{*}$ \\
Kidney & $1.13(0.1)$ & $1.38(0.1)$ & & $1.12(0.2)$ & $1.49(0.2)$ \\
\hline
\end{tabular}

Results are given as mean (SE) for mRNA expression (fold change compared to reference controls)

${ }^{*} p<0.01$ and ${ }^{* *} p<0.001$ vs control rats. 
Figure 3 Representative western blots for vascular endothelial growth factor-A (VEGF-A) in the liver, mesentery and pancreas of three control (CT) and three cirrhotic rats with ascites $(\mathrm{CH}+\mathrm{A})$. Densitometric analysis of all samples is shown in the bottom right panel.
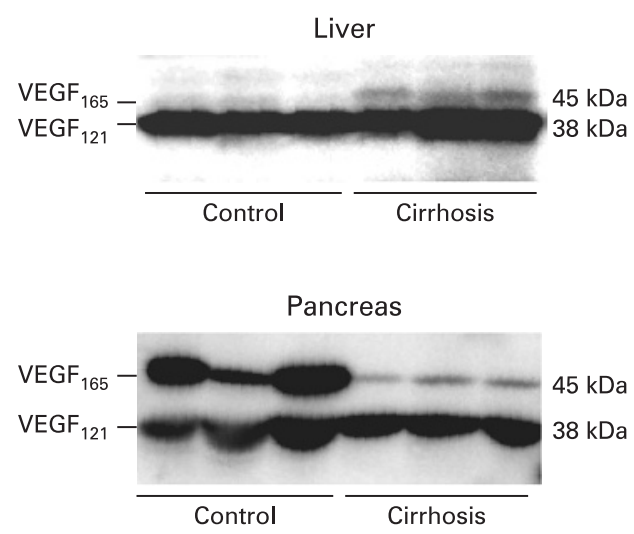
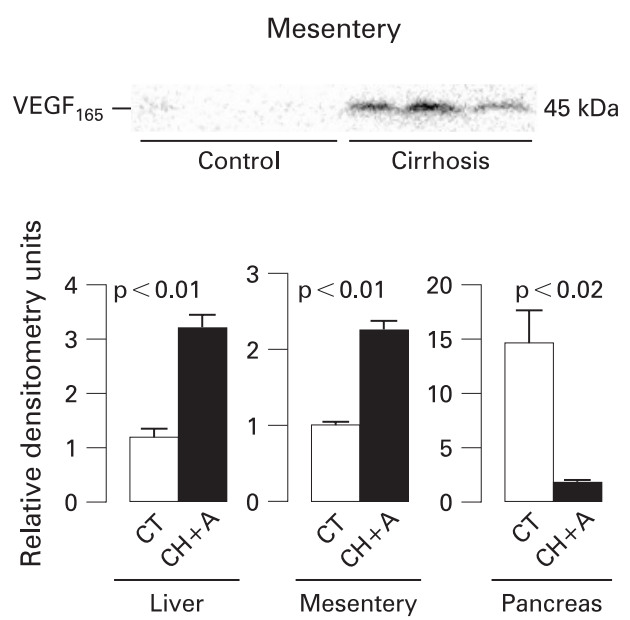

isoform. The protein signal of the $\mathrm{VEGF}_{121}$ isoform was similar in both groups of animals. The densitometric analysis of VEGFA expression in the mesentery delivers qualitatively similar conclusions, indicating increased abundance of VEGF-A in cirrhotic animals. Conversely, western blot analysis of VEGF$A$ in pancreatic homogenates resulted in an reduced expression of this substance, thus mirroring the results found when vascular permeability was assessed.

As shown in fig 4, the protein content of Ang-2 in the different organs yielded very similar results to those obtained with VEGF-A. Actually, the liver and mesentery of cirrhotic rats showed enhanced abundance of Ang-2, whereas an acute reduction of this peptide was noted in the pancreas of these animals in comparison to controls. Moreover, and in agreement with the results obtained on assessing mRNA expression, the protein abundance of Ang-2 was significantly higher in the small intestine than in controls. The protein content of Ang-1 was also assessed in the liver, mesentery, small intestine and pancreas of control and cirrhotic rats. In agreement with previous findings obtained in our laboratory, ${ }^{23}$ all these organs, except the pancreas, showed increased abundance of Ang-1 in cirrhotic rats with ascites compared with control animals (fig 5).

In an attempt to identify the cellular source of the altered expression of vascular permeability factors, we performed histological immunolocalisation of VEGF-A and Ang-2 in liver and pancreas of cirrhotic and control rats. VEGF-A and Ang-2 immunolabelling was only detected in the first row of perivenular hepatocytes of control rats. In cirrhotic animals, most hepatocytes appeared as VEGF-A and Ang-2 positive cells and no signal was detected in bile duct epithelial cells and myofibroblasts (figs 6 and 7, respectively). Expression of VEGF$A$ and Ang-2 in the pancreas of control rats was restricted to cells located in the pancreatic islets of control animals and this signal almost disappeared on examining pancreatic sections from cirrhotic rats (figs 6 and 7, respectively).

Effect of VEGFR $\mathbf{2}_{\mathbf{2}}$ blockade on vascular leakage in cirrhotic rats In a further attempt to establish a direct relationship between the increased expression of vascular permeability factors and altered tissue leakage in liver disease we assessed the effect of acutely blocking VEGFR 2 on vascular permeability in cirrhotic rats with ascites. In vivo efficacy of SU11248 was assessed by comparing hepatic VEGFR 2 phosphorylation between treated and untreated cirrhotic animals. As shown in supplementary fig 1, VEGFR 2 blockade almost suppressed the presence of the phosphorylated form of this receptor in the liver of cirrhotic rats. The administration of the $\mathrm{VEGFR}_{2}$ inhibitor markedly reduced vascular hyperpermeability in the liver and mesentery of these animals. In fact, extravasation of Evan's Blue in these organs was 3- and 2-fold lower in cirrhotic rats receiving SU11248 than in those treated with vehicle (table 3).

\section{DISCUSSION}

The results of the current investigation indicate that hyperpermeabilty selectively occurs in the liver and the mesenteric vasculature, but not in the spleen, pancreas, small intestine or kidney of cirrhotic rats. This is accompanied by a strong positive signal for VEGF-A and Ang-2 in hepatic parenchymal cells and paralleled by enhanced messenger and protein expression of
Figure 4 Representative western blots for angiopoietin-2 (Ang-2) in the liver, small intestine, pancreas and mesentery of three control (CT) and three cirrhotic rats with ascites $(\mathrm{CH}+\mathrm{A})$. Densitometric analysis of all samples is shown in the bottom right panel.
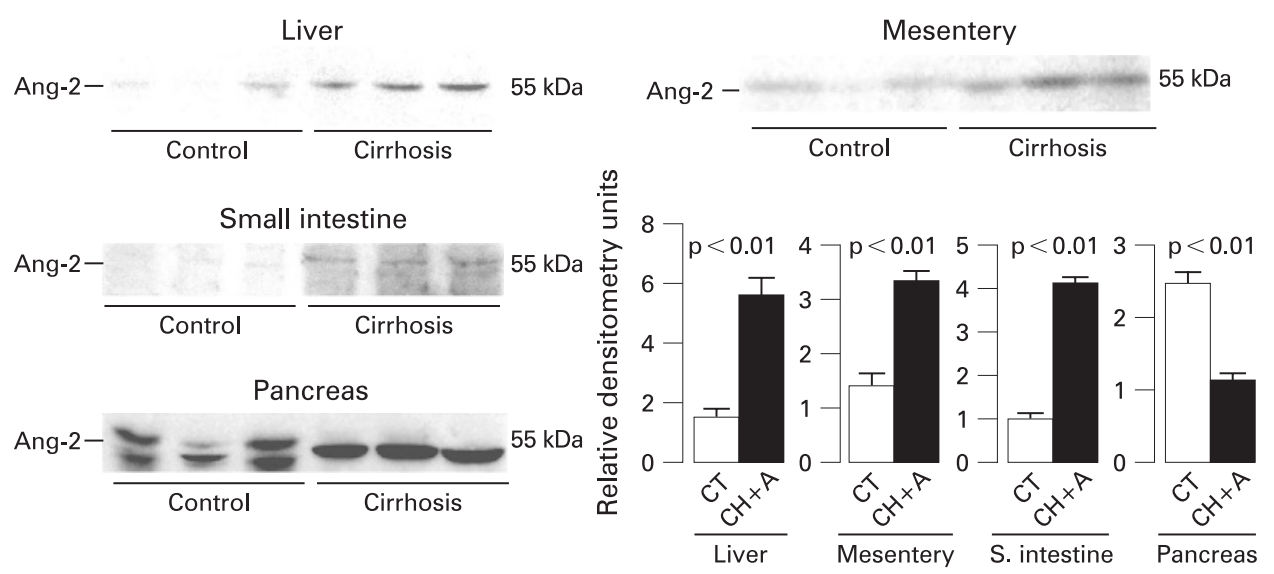
Figure 5 Representative western blots for angiopoietin-1 (Ang-1) in the liver, small intestine pancreas and mesentery of three control (CT) and three cirrhotic rats with ascites $(\mathrm{CH}+\mathrm{A})$. Densitometric analysis of all samples is shown in the bottom right panel.

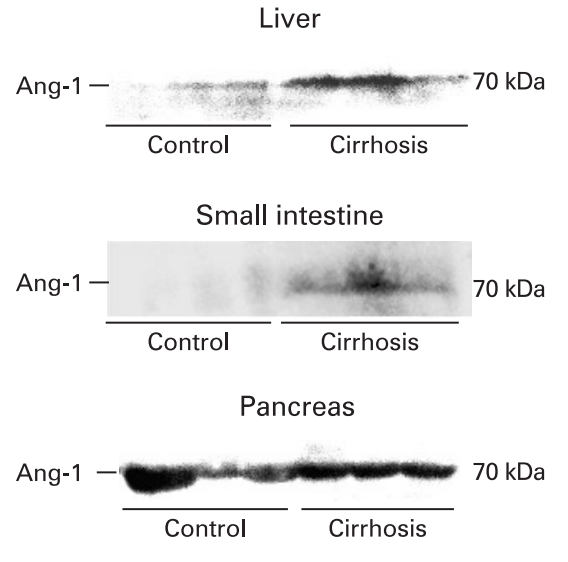

these vascular permeability factors in both, liver and mesentery of cirrhotic animals. Moreover, acute $\mathrm{VEGFR}_{2}$ blockade reduces hepatic and mesenteric vascular permeability to nearly normal values in cirrhotic rats. Altogether, these results indicate that both VEGF-A and Ang-2 are involved in vascular hyperpermeabiliy and the formation of ascites in experimental cirrhosis.

Histological examination of local leakage using colloidal carbon confirmed that the cirrhotic liver and the mesentery are hyperpermeable to macromolecules. In normal livers, the ink was only found within the sinusoids, thus reflecting the architectural integrity of the hepatic tissue. Narrowing and collagenisation of the hepatic sinusoids were associated with a strong presence of the marker close to the fibrous septa. Previous studies have demonstrated that these areas are surrounded by a dense and relatively immature vasculature, ${ }^{23} 24$ thus our results indicate that the increased vascular leakage in the cirrhotic liver is a consequence of the previously described angiogenic processes associated with fibrogenesis. ${ }^{23}$ Accordingly, it is very likely that our observation of enhanced vascular permeability in the mesenteric vasculature is also related to the existence of ongoing angiogenesis in this territory. This would be consistent with recent investigations demonstrating increased abundance of CD31, a marker of endothelial cells, in the mesentery of rats with portal hypertension as compared to control rats. ${ }^{25}$ Conversely, the pancreas of cirrhotic animals showed markedly decreased vascular permeability in comparison to control rats. This finding was associated with impaired vascularisation and decreased expression of VEGF-A and Ang-2. As far we know, this is the first study describing diminished pancreatic vessel density in experimental cirrhosis. Since vascularisation of the pancreatic tissue is highly dependent on proangiogenic signalling pathways ${ }^{26}$ it is possible that disruption of these regulatory mechanisms by inflammatory substances produced in response to the hepatic injury ${ }^{3}$ result in the islet vascularisation and permeability abnormalities observed in cirrhotic rats. Furthermore, this could be a new mechanism involved in the frequent impaired islet insulin output of patients with liver disease.
Figure 6 Representative images corresponding to the immunohistochemistry for vascular endothelial growth factor-A (VEGF-A) in the liver and pancreas of control rats and cirrhotic rats with ascites.
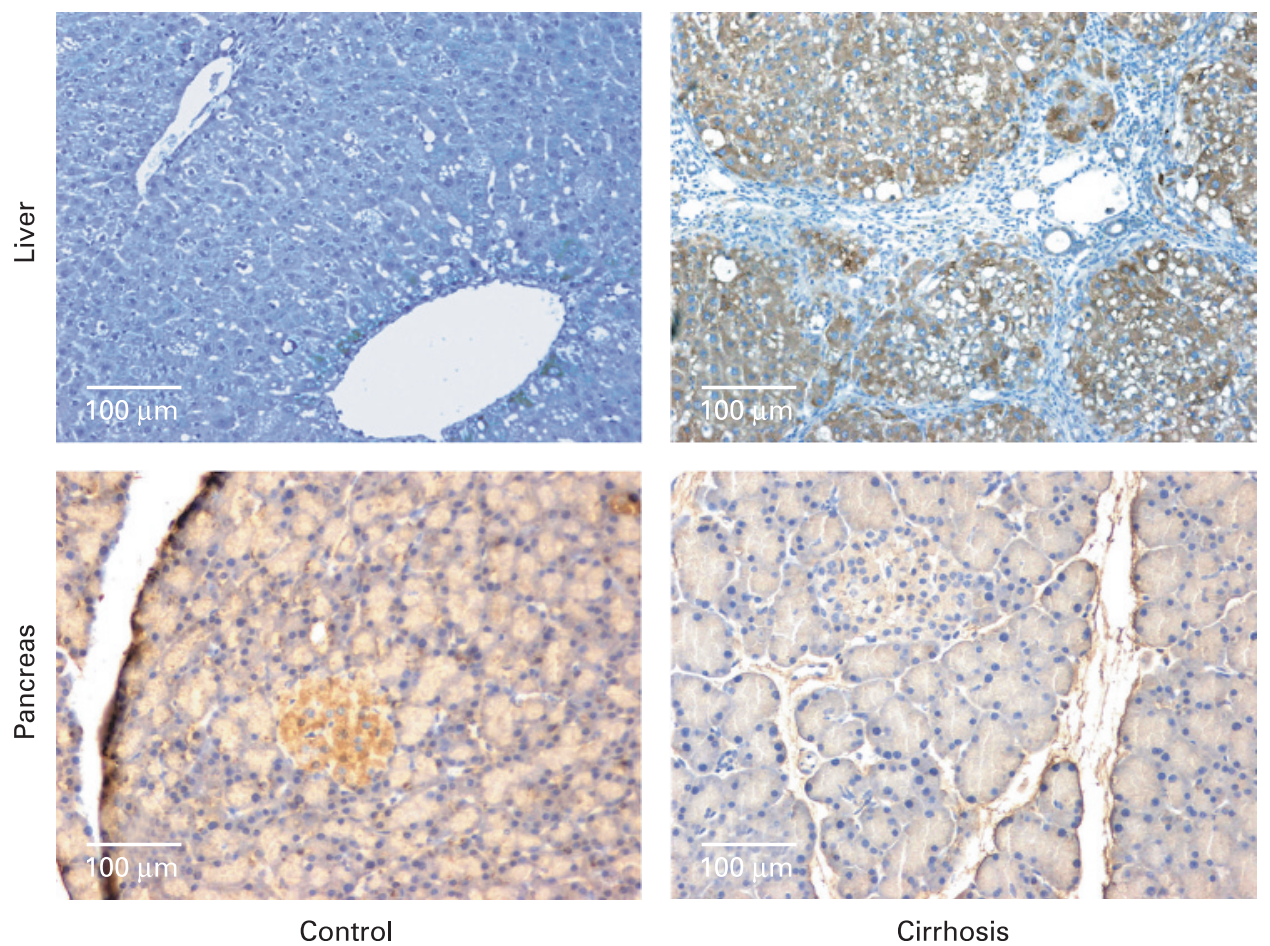

Cirrhosis 
Figure 7 Representative images corresponding to the immunohistochemistry for angiopoietin-2 (Ang-2) in the liver and pancreas of control rats and cirrhotic rats with ascites.
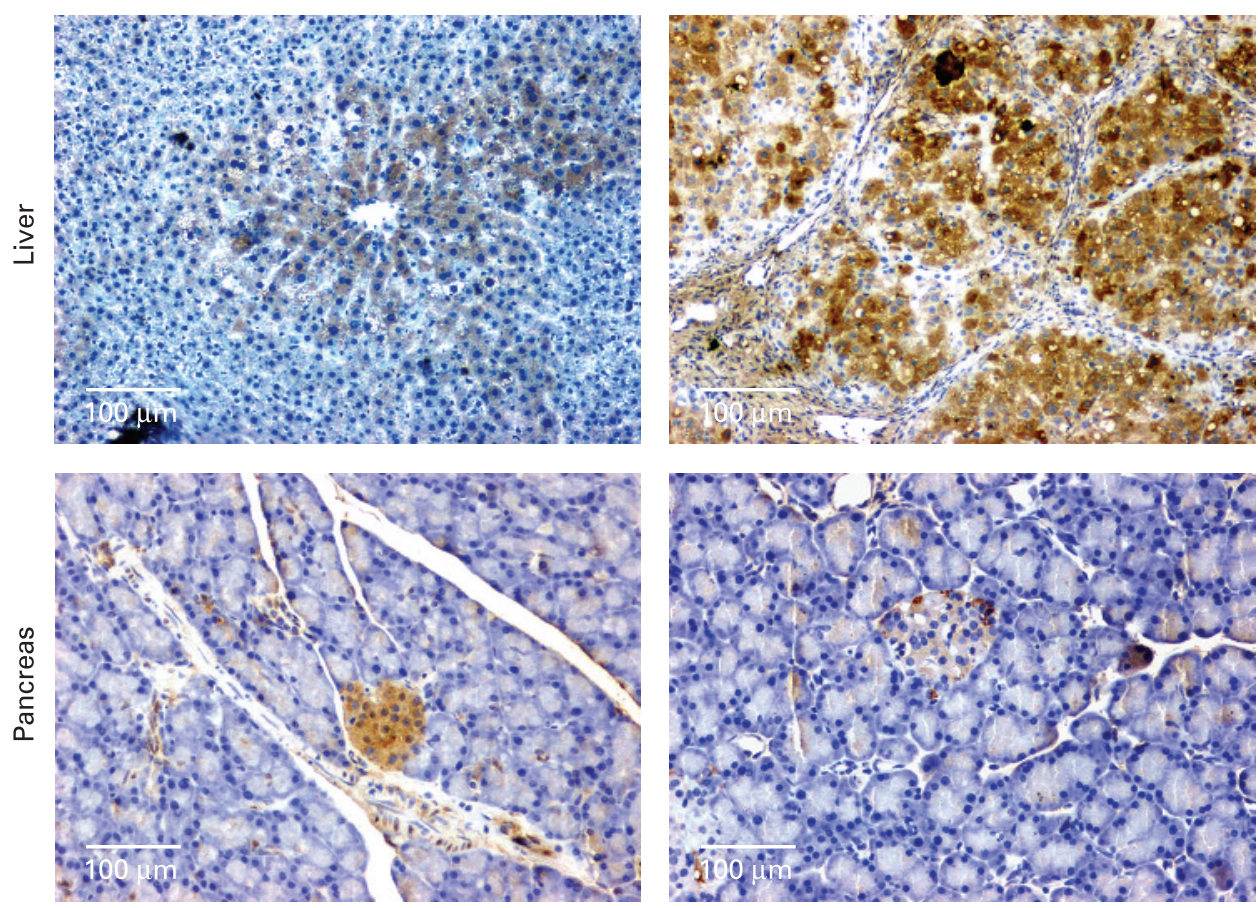

Control

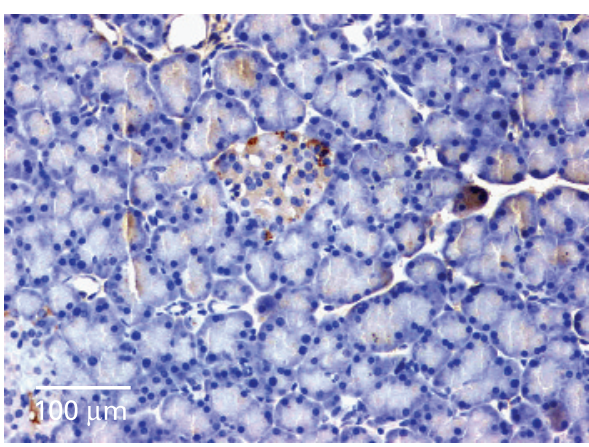

Cirrhosis
It is widely accepted that alterations in blood flow exert a direct influence on microvascular permeability. ${ }^{27}$ Since cirrhotic animals displayed increased total blood flow in the organs that showed enhanced vascular permeability, the contributory role of the hepatic and mesenteric hyperperfusion to the excessive leakage observed in these territories cannot be excluded. However, this is not the sole factor explaining this phenomenon. In fact, pancreas and small intestine of cirrhotic animals showed enhanced total blood flow but reduced, or unaltered vascular extravasation, respectively.

We found that mRNA and protein expression of the permeability factors VEGF-A and Ang-2 rose in the liver and the mesentery, but not in the spleen, pancreas, small intestine and kidney of cirrhotic rats. Indeed, the former were those showing significantly increased permeability values. Immunolocalisation experiments revealed a strong positive signal for both factors throughout the hepatic parenchyma of cirrhotic animals. This probably results from the extensive hypoxic areas previously described in the cirrhotic liver. ${ }^{23} 28$ Interestingly, we also found VEGF-A and Ang-2 expression in normal hepatic tissue albeit restricted to the perivenular hepatocytes, thus reflecting the lower partial pressure of oxygen $\left(\mathrm{PO}_{2}\right)$ surrounding these cells.

Table 3 Effect of vascular endothelial growth factor receptor 2 $\left(\mathrm{VEGFR}_{2}\right.$ ) blockade on vascular leakage in cirrhotic rats with ascites

\begin{tabular}{lcc}
\hline Tissue & Cirrhotic rats $(\mathbf{n}=\mathbf{5})$ & Cirrhotic rats + SU11248 $(\mathbf{n}=\mathbf{5})$ \\
\hline Liver & $148.2(20.9)$ & $59.7(5.3)^{* *}$ \\
Mesentery & $13.4(2.2)$ & $6.0(1.0)^{*}$ \\
Pancreas & $10.1(2.9)$ & $17.9(3.6)$ \\
Spleen & $120.4(12.4)$ & $155.0(21.2)$ \\
Small intestine & $17.1(2.9)$ & $14.9(2.5)$ \\
Kidney & $118.3(27.9)$ & $65.3(23.0)$
\end{tabular}

Results (mean (SE)) are given for the regional vascular permeability ( $\mu \mathrm{g}$ Evan's Blue per $\mathrm{g}$ tissue).

${ }^{*} \mathrm{p}<0.01$ and ${ }^{* *} \mathrm{p}<0.001$ vs control rats.
These data suggest a cooperative and dependent role for both, VEGF-A and Ang-2, in the regulation of vascular permeability in the liver and mesentery of cirrhotic animals. These two factors are critical to explain vascular leakage in the cirrhotic tissues. Both are expressed in response to hypoxia and VEGF-A possesses a potent ability to permeate capillaries to a level 50000 -fold higher than histamine. ${ }^{29}$ Ang-2 is a receptor ligand acting on endothelial cells which allows vascular remodelling by antagonising angiopoietin-1 (Ang-1), a member of the same family of molecules. ${ }^{30}$ Ang-1 induces capillary maturation which then becomes quiescent and unresponsive to VEGF-A action unless Ang-2 is also secreted. ${ }^{31}$ Hence, Ang-2 is an endogenous Ang-1 antagonist that displaces Ang-1 from its receptor and induces vessel destabilisation. ${ }^{14}$ Consequently, in a scenario in which Ang- 1 is activated, as occurs in the liver and mesentery of cirrhotic rats, the concomitant activation of Ang-2 is a critical event allowing vessel remodelling upon VEGF-A action. This is in line with our findings showing that upregulation of Ang-1 and Ang-2, but not VEGF, does not result in vascular hyperpermeability in the intestine of cirrhotic animals.

Endothelial cells exposed to VEGF-A allow passage of small solutes by inducing the formation of fenestration and/or caveolae. ${ }^{32}{ }^{33}$ Leakage of larger proteins likely occurs between adjacent endothelial cells. ${ }^{10}$ Disruption of cell-to-cell junctional integrity results in extravasation of serum/plasma proteins, leading to accumulation of fluids in the extracellular space. VEGFR $_{2}$ mediates regulation of junctional integrity and constitutes a major mechanism for localised disruption by VEGF-A. ${ }^{10}$ SU11248 is an indolinone molecule which has been shown to possess potent antiangiogenic properties owing to its selective inhibition of $\mathrm{VEGFR}_{2}$ and platelet derived growth factor receptor- $\beta$ (PDGFR- $\beta$ ) phosphorylation. ${ }^{34}$ This tyrosine kinase inhibitor appears to be a powerful tool for elucidating the contribution of VEGF-A activation to the increased vascular permeability in cirrhosis since PDGFR- $\beta$ is related to functions other than regulation of vascular extravasation, including pericyte recruitment and maturation of blood capillaries. ${ }^{14}$ In 
fact, acute inhibition of $\mathrm{VEGFR}_{2}$ phosphorylation markedly reduced vessel permeability in the liver and mesentery of cirrhotic rats with ascites, further emphasising the importance of this signalling pathway in the pathogenesis of vascular leakage in experimental cirrhosis.

Funding: This work was supported by grants from Dirección General de Investigación Científica y Técnica (SAF03-02597 and SAF06-07053 to WJ, and SAF07-63069 to MM-R). PM-L had a grant from DGICYT (BES-2004-5186). ST had a grant from IDIBAPS. GF-V had a clinical chemistry fellowship from Siemens Medical Solutions Diagnostics (Tarrytown, New York, USA). SU11248 was generously supplied by Pfizer, South San Francisco, California, USA. CIBERehd is funded by the Instituto de Salud Carlos III, Spain.

Competing interests: None declared.

Ethics approval: Approval for this study was obtained from the Investigation and Ethics Committees of the Hospital Clinic on 17 December 2002.

\section{REFERENCES}

1. Arroyo V, Ginés $P$, Jiménez W, et al. Renal dysfunction in cirrhosis. In: Bircher J, Benhamou JP, Mclntyre N, et al, eds. Oxford textbook of clinical hepatology, 2nd edn. Oxford: Oxford Medical Publications, 1999: 733-61.

2. Laffi G, Foschi M, Masini E, et al. Increased production of nitric oxide by neutrophils and monocytes from cirrhotic patients with ascites and hyperdynamic circulation. Hepatology 1995;22:1666-73.

3. Navasa M, Follo A, Filella $X$, et al. Tumor necrosis factor and interleukin-6 in spontaneous bacterial peritonitis in cirrhosis: relationship with the development of renal impairment and mortality. Hepatology 1998;27:1227-32.

4. Jiménez W, Ros J, Morales-Ruiz $\mathrm{M}$, et al. Nitric oxide production and inducible nitric oxide synthase expression in peritoneal macrophages of cirrhotic patients. Hepatology 1999:30:670-6.

5. Cejudo-Martin P, Morales-Ruiz M, Ros J, et al. Hypoxia is an inducer of vasodilator agents in peritoneal macrophages of cirrhotic patients. Hepatology 2002;36:1172-9.

6. Pérez-Ruiz M, Ros J, Morales-Ruiz M, et al. Vascular endothelial growth factor production in peritoneal macrophages of cirrhotic patients: regulation by cytokines and bacterial lipopolysaccharide. Hepatology 1999;29:1057-63.

7. Morales-Ruiz M, Tugues S, Cejudo-Martin P, et al. Ascites from cirrhotic patients induces angiogenesis through the phosphoinositide 3-kinase/Akt signaling pathway. J Hepatol 2005;43:85-91.

8. Hyatt RE, Smith JR. The mechanism of ascites, a physiologic appraisal. Am J Med 1954;16:434-8.

9. Levy M, Wexler MJ. Renal sodium retention and ascites formation in dogs with experimental cirrhosis but without portal hypertension or increased splanchnic vascular capacity. J Lab Clin Med 1978;91:520-36.

10. Weis S M, Cheresh DA. Pathophysiological consequences of VEGF-A induced vascular permeability. Nature 2005:437:497-504.

11. Yancopoulos GD, Davis S, Gale NW, et al. Vascular specific growth factors and blood vessel formation. Nature 2000:407:242-8.

12. Morales-Ruiz M, Jiménez W. Neovascularization, angiogenesis, and vascular remodeling in portal hypertension. In: Sanyal AJ, Shah VH, eds. Portal hypertension. Pathogenesis, evaluation and treatment, 1st edn. Totowa: Humana Press, 2005: 99-112.

13. Roviezzo F, Tsigkos S, Kotanidou A, et al. Angiopoietin-2 causes inflammation in vivo by promoting vascular leakage. J Pharmacol Exp Ther 2005;314:738-44.

14. Pouyssegur J, Dayan F, Mazure NM. Hypoxia signalling in cancer and approaches to enforce tumour regression. Nature 2006;441:437-43.
15. Clària J, Jiménez W. Renal dysfunction and ascites in carbon-tetrachloride-induced cirrhosis in rats. In: Ginés P, Arroyo V, Rodès J, Schrier RW, eds. Ascites and renal dysfunction in liver disease. Pathogenesis diagnosis and treatment, 2nd edn. Malden, MA: Blackwell Science, 2005: 215-26.

16. Rogers DF, Boschetto P, Barnes PJ. Plasma exudation. Correlation between Evans blue dye and radiolabeled albumin in guinea pig airways in vivo. J Pharmacol Methods 1989:21:309-15

17. Nagy JA, Masse EM, Herzberg KT, et al. Pathogenesis of ascites tumor growth: vascular permeability factor, vascular hyperpermeability, and ascites fluid accumulation. Cancer Res 1995;55:360-8.

18. Lortie M, Sirois MG, Couture R, et al. Regional changes in the extravasation of albumin in the canine kidney: comparison of bradykinin and water diuresis. Microvasc Res 1994; 48:282-94

19. Dvorak HF, Nagy JA, Dvorak JT, et al. Identification and characterization of the blood vessels of solid tumors that are leaky to circulating macromolecules. Am J Pathol 1988; 133:95-109

20. Yuan Y, Granger HJ, Zawieja DC, et al. Flow modulates coronary venular permeability by a nitric oxide-related mechanism. Am J Physiol 1992;263:H641-6.

21. Bosch J, García-Pagan JC. The splanchnic circulation in cirrhosis. In: Ginés P, Arroyo V. Rodés J, Schrier RW, eds. Ascites and renal dysfunction in liver disease, 2nd edn. Malden: Blackwell Publishing, 2005: 156-63.

22. Bosch J, García-Pagan JC. Complications of cirrhosis. I. Portal hypertension. $J$ Hepatol 2000;32:141-56.

23. Tugues S, Fernández-Varo G, Muñoz-Luque J, et al. Antiangiogenic treatment with sunitinib ameliorates inflammatory infiltrate, fibrosis, and portal pressure in cirrhotic rats. Hepatology 2007:46:1919-26.

24. Rappaport AM, MacPhee PJ, Fisher MM, et al. The scarring of the liver acini (cirrhosis). Tridimensional and microcirculatory considerations. Vinchows Arch A Pathol Anat Histopathol 1983;402:107-37

25. Fernández M, Mejías M, Angermayr B, et al. Inhibition of VEGF receptor-2 decreases the development of hyperdynamic splanchnic circulation and portal-systemic collateral vessels in portal hypertensive rats. J Hepatol 2005;43:98-103.

26. Brissova M, Shostak $A$, Shiota $M$, et al. Pancreatic islet production of vascular endothelial growth factor-A is essential for islet vascularization, revascularization, and function. Diabetes 2006;55:2974-85.

27. Sarelius IH, Kuebel JM, Wang J, et al. Macromolecule permeability of in situ and excised rodent skeletal muscle arterioles and venules. Am J Physiol Heart Circ Physiol 2006;290:H474-80.

28. Rosmorduc 0, Wendum D, Corpechot C, et al. Hepatocellular hypoxia-induced vascular endothelial growth factor expression and angiogenesis in experimental biliary cirrhosis. Am J Path 1999;155:1065-73.

29. Senger DR, Galli SJ, Dvorak AM, et al. Tumors cells secrete a vascular permeability factor that promotes accumulation of ascites fluid. Science 1983:219:983-5.

30. Maisonpierre PC, Suri C, Jones PF, et al. Angiopoietin-2, a natural antagonist for Tie2 that disrupts in vivo angiogenesis. Science 1997;277:55-60.

31. Noseda M, Chang L, McLean G, et al. Notch activation induces endothelial cell cycle arrest and participates in contact inhibition: role of p21Cip1 repression. Mol Cell Biol 2004:24:8813-22.

32. Roberts WG, Palade GE. Increased microvascular permeability and endothelia fenestration induced by vascular endothelial growth factor. J Cell Sci 1995; 108:2369-79.

33. Esser S, Wolburg $\mathrm{K}$, Wolburg $\mathrm{H}$, et al. Vascular endothelial growth factor induces endothelial fenestrations in vitro. J Cell Biol 1998:140:947-59.

34. Mendel DB, Laird AD, Xin X, et al. In vivo antitumor activity of SU11248, a nove tyrosine kinase inhibitor targeting vascular endothelial growth factor and plateletderived growth factor receptors: determination of a pharmacokinetic/ pharmacodynamic relationship. Clin Cancer Res 2003;9:327-37. 